\title{
An integrated emergency department/hospital at home model in mild COVID-19 pneumonia: feasibility and outcomes after discharge from the emergency department: comment
}

\author{
Giovanni Nattino $^{1}$ (D) Stefano Paglia ${ }^{2}$
}

Received: 20 March 2021 / Accepted: 23 March 2021 / Published online: 17 April 2021

(c) Società Italiana di Medicina Interna (SIMI) 2021

We congratulate with Llorens and colleagues for their interesting paper, titled "An integrated emergency department/ hospital at home model in mild COVID-19 pneumonia: feasibility and outcomes after discharge from the emergency department" [1]. The implemented home hospitalization intervention for COVID-19 patients directly addresses the shortage of hospital beds and the related emergency department (ED) crowding, which have been observed worldwide during the pandemic. Specifically, in the EDs of the areas interested by COVID-19 outbreaks, the imbalance between the number and needs of the patients visiting the EDs (input) and the limited availability of COVID-19-dedicated hospital beds (output) have caused the dramatic increase of boarding times [2]. In this context, the possibility to discharge at home the ED patients with mild symptoms of recent onset who would have been alternatively hospitalized and to provide them with appropriate monitoring care pathways is a first effective strategy to protect the finite hospital resources. In particular, telemonitoring systems offer the possibility to remotely monitor vital signs and symptoms of patients at home, to promptly identify clinical deteriorations. Hospital-at-home models, such as the one implemented by the authors, involve acute care of patients and include administering treatments and performing diagnostic tests, in addition to a continuous surveillance and evaluation of the patients' needs.

To identify the patients who can be safely enrolled in homecare programs, tests with high sensitivity for the

Giovanni Nattino

giovanni.nattino@marionegri.it

Stefano Paglia

stefano.paglia@asst-lodi.it

1 Laboratory of Clinical Epidemiology, Istituto di Ricerche Farmacologiche Mario Negri IRCCS, Ranica, BG, Italy

2 Emergency Department, ASST Lodi, Lodi, LO, Italy identification of patients at risk of rapid deteriorations are deemed essential. In this context, exercise testing has been variously used in the evaluation of COVID-19 patients, with different aims and approaches. Among the criteria to enroll patients into the hospital-at-home program, LLorens and colleagues used a 50-m walk test, considered as negative in case of a pulse oximeter saturation $>93 \%$ after the exercise and a difference between pre- and post-exercise saturation smaller than 5\%. Previous research has found a limited prognostic value of post-exercise saturation for poor outcomes (30-day death or need of organ support) in a cohort including all COVID-19 ED patients [3]. However, for those patients with minor pulmonary dysfunctions, i.e., those patients who would be eligible to homecare programs, a quick walk test was found to have high sensitivity and specificity for shortterm need of mechanical ventilation [4]. In light of these results, considering the low rate of deferred hospital admissions in the study by LLorens and colleagues, with no ICU admission, and that such tests can be performed easily and quickly in emergency settings, with no specialized equipment and by nonmedical personnel, exercise tests could be very useful in the stratification and monitoring of COVID-19 patients, even if a definitive validation is still needed.

The possibility to discharge eligible COVID-19 patients from the ED and provide adequate care at home may be an effective strategy to reduce the need for COVID-19-dedicated hospital beds and, consequently, ED boarding times. However, the beneficial effects of homecare models could be maximized by an early, territorial assessment, anticipating the possible decision of visiting an ED. The benefits would be twofold. On the one hand, patients at risk of rapid deteriorations could be effectively monitored and promptly treated. On the other hand, such assessments would defer the presentation of inappropriate visits of paucisymptomatic patients, reducing ED overcrowding and waiting times. Nevertheless, to implement appropriate territorial assessments in practice, a concerted coordination of regional and national health 
systems with territorial home care services is essential, to guarantee the availability of appropriate infrastructure and medical resources. Minimal needs include the possibility to perform point-of-care lung ultrasound tests [5] and monitor blood saturation with pulse oximeters [6], possibly applying walk tests. Moreover, effective programs must necessarily involve key actors of community-based healthcare services, such as family practitioners, rehabilitation physicians and physiotherapists, and should facilitate their communication with ED operators.

In the context of progressive demographic and social transitions toward older ages and higher prevalence of chronic diseases [7], the COVID-19 pandemic has exacerbated longknown problems of hospital-centered health systems lacking of strong home-based care services [8]. Even in high-income countries, investments in homecare services have been very heterogeneous. In Europe, for example, the number of family practitioners per 100.000 inhabitants in 2018 spanned from 41 in Greece to 269 in Portugal, a sixfold difference [9]. With the perspective of addressing the shortcomings of healthcare systems in the post-COVID era, key foundational conditions have been advocated for the worldwide primary healthcare systems [10]. Such systems should move towards integrated, comprehensive, interdisciplinary approaches, characterized by a continuum between hospital and local services, centered on people's health, embrace territorialized interventions based on specific health needs and be orientated towards prevention and health promotion rather than the provision of individual services. As the dramatic consequences of the COVID-19 pandemic have drawn attention to the flaws of our homecare health systems, this is the moment to keep the momentum and trigger the implementation of the necessary policy changes, avoiding arbitrary choices and using appropriate research designs to measure effectiveness and efficacy of the interventions.

Author contributions Both authors drafted the manuscript and approved the final version.

Funding The authors received no specific funding for this work.

\section{Declarations}

Conflict of interest The authors have no conflict of interest or competing interest to declare.
Ethics approval Not applicable.

Consent to participate Not applicable.

\section{References}

1. Llorens P, Moreno-Pèrez $\mathrm{O}$, Espinosa B et al (2021) An integrated emergency department/hospital at home model in mild COVID19 pneumonia: feasibility and outcomes after discharge from the emergency department. Internal Emerg Med 12:34-56

2. Paglia S, Storti E, Bisagni P et al (2020) Lodi's experience in handling the first COVID-19 hotbed in Europe. Ital J Emerg Med 9:93-101

3. Goodacre S, Thomas B, Lee E et al (2021) Post-exertion oxygen saturation as a prognostic factor for adverse outcome in patients attending the emergency department with suspected COVID-19: a substudy of the PRIEST observational cohort study. Emerg Med J 38:88-93. https://doi.org/10.1136/emermed-2020-210528

4. Paglia S, Nattino G, Occhipinti F et al (2021) The quick walk test: a noninvasive test to assess the risk of mechanical ventilation during COVID-19 outbreaks. Acad Emerg Med 28:244-247. https:// doi.org/10.1111/acem.14180

5. Soldati G, Smargiassi A, Inchingolo R et al (2020) Is there a role for lung ultrasound during the COVID-19 pandemic? J Ultrasound Med 39:1459-1462. https://doi.org/10.1002/jum.15284

6. Shah S, Majmudar K, Stein A et al (2020) Novel use of home pulse oximetry monitoring in COVID-19 patients discharged from the emergency department identifies need for hospitalization. AcadEmerge Med. https://doi.org/10.1111/acem.14053

7. Christensen K, Doblhammer G, Rau R, Vaupel JW (2009) Ageing populations: the challenges ahead. The Lancet 374:1196-1208. https://doi.org/10.1016/S0140-6736(09)61460-4

8. Volpato S, Landi F, Incalzi RA, on behalf of the Italian Society of Gerontology and Geriatrics (2020) A frail health care system for an old population: lesson form the COVID-19 outbreak in Italy. $\mathrm{J}$ Gerontol Series A 75:e126-e127. https://doi.org/10.1093/gerona/ glaa087

9. Eurostat (2018) Healthcare personnel statistics - Physicians. https://ec.europa.eu/eurostat/statistics-explained/index.php?title= Healthcare_personnel_statistics_-_physicians. (Accessed $18 \mathrm{Mar}$ 2021)

10. World Health Organization (2018) From Alma-Ata to Astana: primary health care-reflecting on the past, transforming for the future. WHO

Publisher's Note Springer Nature remains neutral with regard to jurisdictional claims in published maps and institutional affiliations. 